\title{
Qualidade de vida e incidência de depressão em idosas que frequentam grupos de terceira idade
}

\author{
Gleice Helena Jacques Casagrande*, Monique Farias da Silva", Pâmela Billig Mello Carpes"**
}

\section{Resumo}

O objetivo deste estudo foi avaliar a qualidade de vida e a incidência de depressão em idosas que participam de grupos de terceira idade de Uruguaiana - RS e relacionar esses resultados com a sua percepção acerca desses aspectos. Selecionou-se por voluntariedade 42 idosas participantes de grupos de terceira idade de Uruguaiana $\mathrm{RS}$, as quais foram submetidas a avaliações da cognição (Mini-Exame do Estado Mental, MEEM), presença de depressão (Escala Geriátrica de Depressão, EGD-30) e qualidade de vida (SF36), e entrevistadas com o objetivo de verificar sua percepção acerca dos mesmos aspectos. Os resultados quantitativos obtidos foram analisados por meio de estatística descritiva; e as entrevistas foram gravadas e, posteriormente, transcritas para a análise em profundidade. A idade média da amostra foi de 71,3 anos, com tempo médio de participação no grupo de 3,6 anos. O escore médio no MEEM foi de 24,04 pontos, tendo somente sete idosas apresentado pontuação abaixo do ponto de corte. Por meio do EGD-30 verificou-se que $88,1 \%$ das idosas avaliadas não apre-

\begin{abstract}
sentaram sintomas depressivos e 11,9\% apresentaram sintomas de depressão moderada. A média do SF-36 foi de 98,6, o que, somado às ideias percebidas nas falas, evidencia que a maioria das idosas tem boa qualidade de vida geral. Verificou-se que a incidência de depressão e deficit cognitivo entre as idosas avaliadas foi baixa, o que pode estar relacionado com a sua participação em grupos de convivência, conforme enfatizado em suas falas.
\end{abstract}

Palavras-chave: Qualidade de vida. Mini-Mental. Atividade física. Idosos. Terceira idade.

\section{Introdução}

A mudança do perfil demográfico mundial é um tema que tem promovido discussões e investigações, sendo evidenciado o aumento da população idosa no mundo todo. Mendes et al. (2005) des-

* Acadêmica do curso de Fisioterapia da Universidade Federal do Pampa.

** Acadêmica do curso de Fisioterapia da Universidade Federal do Pampa.

**:* Fisioterapeuta pela Universidade de Cruz Alta. Mestra e Doutora em Ciências Biológicas: Fisiologia pela Universidade Federal do Rio Grande do Sul. Professora adjunta da Universidade Federal do Pampa. Líder do Grupo de Pesquisa em Fisiologia (GPFis). Endereço para correspondência: Universidade Federal do Pampa, Laboratório de Estresse, Memória e Comportamento, Campus Uruguaiana, BR 472, km 582, Caixa postal 118, CEP: 97500-970, Uruguaiana - RS. E-mail: pamelacarpes@unipampa.edu.br.

$\rightarrow$ http://dx.doi.org/10.5335/rbceh.2013.2940 
tacam que, no Brasil, estima-se que nos próximos 20 anos o contingente de idosos poderá alcançar e até mesmo ultrapassar 30 milhões de pessoas, o que representará aproximadamente $13 \%$ da população.

A World Health Organization (2005) calcula que, até 2025, o Brasil será o sexto país do mundo com maior número de idosos. Tais transformações têm implicações em todo o ciclo de vida e no perfil das faixas etárias, clamando por novas formas de organização social e políticas condizentes com a sociedade contemporânea. Segundo Ribaric (2012), a longevidade constitui uma característica multifatorial quantitativa que é influenciada por fatores biológicos, ambientais e psicossociais. Dentre todos esses elementos, os fatores de risco modificáveis são especialmente relevantes, porque são passíveis de intervenção.

É consenso que, ao longo do processo de envelhecimento, a manutenção do corpo em atividade revela-se fundamental para conservar as funções e favorecer o melhor desempenho nas atividades da vida diária, uma vez que as transformações que ocorrem no ser humano à medida que envelhece, associadas às mudanças no seu estado de saúde, estilo de vida e nas suas perspectivas, requerem atenção para garantir um envelhecimento com boa qualidade de vida. Conforme Odebrech e Pedroso (2010), o conceito de qualidade de vida é definido como a percepção que o indivíduo tem sobre a sua posição na vida e no contexto de sua cultura, de acordo com os sistemas de valores da sociedade em que se insere e em relação aos seus objetivos, expectativas, padrões e preocupações.
Como afirmam Nascimento et al. (2000), é evidente que costumes saudáveis durante a vida são as melhores garantias não somente para um envelhecimento saudável, como também para um retardamento do processo. São vários os fatores que influenciam o processo de envelhecimento e a qualidade de vida dos idosos, não dependendo apenas do indivíduo o modo como ele irá alcançar o envelhecer saudável. Dentre os principais fatores estão a herança genética, o meio ambiente, a educação, a habitação, o trabalho, a seguridade social, a cultura, o lazer e os hábitos de alimentação.

Nesse sentido, a promoção de um estilo de vida mais ativo na terceira idade tem sido utilizada como estratégia para desenvolver melhora nos padrões de saúde e de qualidade de vida (FERREIRA et al., 2005), e a prática de atividade física tem sido amplamente incentivada, em vista de seus benefícios físicos e psicossociais. (TEIXEIRA; OKUMA, 2004). Hoje, existem fortes evidências de que o idoso que pratica atividade física mostra melhor adaptação orgânica aos esforços físicos, além de maior resistência às doenças e ao estresse. (CASAGRANDE, 2006).

Outro fator crucial para a qualidade de vida na velhice é a construção de uma imagem mais positiva do envelhecimento. Isso pode ser iniciado no ambiente familiar, uma vez que o suporte da família e o convívio com os entes queridos são fatores primordiais para um envelhecimento saudável. Porém, considerando que o idoso enfrenta diversas barreiras no usufruto do lazer, é necessário promover a sua inclusão em diversificadas oportunidades dessa natureza, sendo im- 
portante o incentivo de políticas públicas para construção de espaços apropriados, onde possa desfrutar de ambientes saudáveis e interagir com outras pessoas, trocando experiência e praticando atividades físicas e recreativas.

Os grupos de terceira idade, quando bem estruturados, são um exemplo de espaço onde o idoso tem a oportunidade de conviver com pessoas da mesma faixa etária e realizar atividades físicas e cognitivas. Além disso, a participação nesse tipo de atividade favorece a autoestima, a autonomia, a independência, a autoexpressão e a reinserção social em busca de uma velhice bem-sucedida. (PALMA, 2000).

Pode-se ressaltar que idosos que apresentam vida social intensa têm aumento na qualidade de vida e longevidade. (FRUTUOSO, 1999). Em contrapartida, a ausência de convívio social na velhice pode ser relacionada com a diminuição da capacidade cognitiva (KATZ; RUBIN, 2000), assim como com a depressão. (FREIRE, 2000). Em relação à depressão, além da prevalência de casos em pessoas idosas quando comparadas a jovens, há um predomínio da patologia no gênero feminino, chegando a ser duas vezes mais prevalente na mulher do que no homem, o que pode estar relacionado com a menopausa e/ou com a expectativa de vida maior nesse sexo. (SILVA et al., 2008; MARQUES-TEIXEIRA, 1998).

Assim, considerando o potencial dos grupos de idosos em promover um envelhecimento com melhor qualidade de vida, o objetivo deste estudo foi avaliar a função cognitiva geral, a incidência de depressão e a qualidade de vida de mulheres parti- cipantes de grupos de terceira idade, bem como verificar suas percepções acerca da qualidade de vida e do envelhecimento. A hipótese deste trabalho é que, apesar de as incidências mais altas de depressão em idosos serem em mulheres e da relação da doença com uma pior qualidade de vida, a participação em grupos de terceira idade, com práticas de atividades físicas, cognitivas e convívio social, contrapõe essa tendência. Dessa forma, hipotetizamos que as idosas avaliadas apresentarão baixos índices de depressão, uma boa qualidade de vida e uma percepção positiva acerca de tais aspectos, provavelmente ligada, em grande medida, à sua participação no grupo.

\section{Materiais e métodos}

A amostra do estudo foi composta por 42 mulheres idosas, com mais de 60 anos, participantes de grupos de terceira idade no município de Uruguaiana - RS. Os grupos realizam encontros semanais com atividades recreativas diversas, tais como atividades físicas, musicoterapia $\mathrm{e}$ atividades cognitivas.

Foram critérios de inclusão neste estudo: ter mais de 60 anos, ser do sexo feminino, participar de um grupo de terceira idade e residir em Uruguaiana - RS. Os critérios de exclusão foram assim definidos: ter diagnóstico médico de demência e/ou estar participando de um grupo de terceira idade há menos de 3 meses, por se entender que um tempo muito curto de participação pode ainda não representar o mínimo de envolvimento necessário para haver algum efeito/ benefício. Esta pesquisa foi aprovada pelo 
Comitê de Ética em Pesquisa da Universidade Federal do Pampa (Unipampa) em 10/07/2012 (Parecer no 54.689).

Cada voluntária disposta a participar do estudo passou por uma entrevista para coleta de dados gerais e esclarecimento dos objetivos e procedimentos do protocolo de estudo, assim como para obtenção da assinatura do Termo de Consentimento Livre e Esclarecido. Após a entrevista inicial, as idosas foram submetidas a uma avaliação das funções relacionadas aos objetivos do estudo (cognição, depressão, qualidade de vida e percepção sobre esses aspectos), cujo fluxograma está ilustrado na Figura 1.

Figura 1. Fluxograma de avaliação das idosas

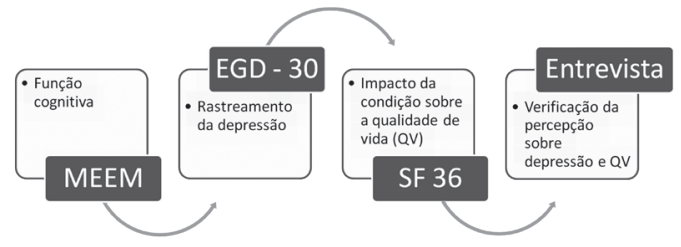

O protocolo de avaliação incluiu os seguintes instrumentos:

a) Mini-Exame do Estado Mental (MEEM): dividido em cinco sessões (orientação, memória imediata, atenção e cálculo, evocação e linguagem), permite a avaliação global da cognição do idoso. O escore máximo possível é de 30 pontos, sendo o idoso classificado de acordo com a escolaridade. Para a classificação, foram considerados os pontos de corte recomendados por Kochhann et al. (2010) para a população do Sul do Brasil, sendo apontadas como idosas com sintomas de deficit cog- nitivo as que obtiveram menos de 21 pontos no caso de analfabetas, 22 no caso de baixa escolaridade ( 1 a 5 anos), 23 para média escolaridade (6 a 11 anos) e 24 para alta escolaridade (acima de 12 anos).

b) Escala Geriátrica de Depressão (EGD-30): A EGD-30 (YESAVAGE et al., 1983) é um dos instrumentos mais frequentemente utilizados para o rastreamento de depressão em idosos e contém 30 itens. As perguntas têm sim e não como opções de resposta, simplicidade que permite que a escala seja utilizada com indivíduos doentes ou com comprometimento cognitivo moderado. Um ponto é atribuído a cada resposta, e a pontuação acumulada é classificada de forma que escores acima de 10 estão relacionados à depressão, sendo considerado moderadamente deprimido o idoso que apresentar pontuação entre 11 e 19, e gravemente deprimido o que apresentar escore de 20 a 30.

c) SF 36: questionário composto por 36 perguntas que avaliam a percepção de uma doença/um evento pelo ponto de vista do próprio sujeito, estando centrado no impacto da condição (envelhecimento) sobre a qualidade de vida, e não na patologia ou nos sinais clínicos, o que constitui o diferencial de tal instrumento. Esse questionário, que foi traduzido e validado no Brasil em 1997 (CAMPOLINA; CICONELLI, 2008), avalia aspectos da qualidade de vida diretamente relacionados à saúde do indivíduo, 
sendo organizado em oito dimensões de saúde: "capacidade funcional", "aspecto físico", "dor", "estado geral de saúde", "vitalidade", "aspectos sociais", "aspectos emocionais" e "saúde mental". A pontuação total do questionário pode chegar a 111 pontos, e, posteriormente, podem ser calculadas notas que variam de 0 a 100 para cada domínio, por meio da seguinte fórmula, sendo fixos os valores expressos na Tabela 1:

Tabela 1. Valores fixos para cálculo da pontuação do SF36

\begin{tabular}{lrr}
\hline \multicolumn{1}{c}{ Domínio } & Limite inferior & Variação \\
\hline Capacidade funcional & 10 & 20 \\
Limitação por aspectos físicos & 4 & 4 \\
Dor & 2 & 10 \\
Estado geral de saúde & 5 & 20 \\
Vitalidade & 4 & 20 \\
Aspectos sociais & 2 & 8 \\
Limitação por aspectos emocionais & 3 & 3 \\
Saúde mental & 5 & 25 \\
\hline
\end{tabular}

Nota: Valor obtido nas questões correspondentes ao domínio - Limite inferior x 100 Variação

d) Entrevista em profundidade: foi realizada uma entrevista em profundidade com as idosas. A entrevista semiestruturada continha seis questões abertas norteadoras que foram construídas pelos pesquisa- dores de acordo com os objetivos do estudo e validadas por pesquisadores da área. As questões utilizadas na entrevista e o objetivo de cada uma são apresentadas no Quadro 1.

Quadro 1. Questões utilizadas na entrevista semiestruturada

\begin{tabular}{|c|c|}
\hline Perguntas da entrevista & Objetivo da questão \\
\hline O que você entende por qualidade de vida? & $\begin{array}{l}\text { Verificar a concepção de qualidade de vida que o } \\
\text { entrevistado possui. }\end{array}$ \\
\hline $\begin{array}{l}\text { Que fatores você acha que influenciam mais na sua } \\
\text { qualidade de vida? }\end{array}$ & $\begin{array}{l}\text { Verificar que fatores os entrevistados relacionam como } \\
\text { influentes na sua qualidade de vida. }\end{array}$ \\
\hline Há quanto tempo você participa de um grupo de idosos? & $\begin{array}{l}\text { Verificar há quanto tempo o idoso participa de um } \\
\text { grupo. }\end{array}$ \\
\hline $\begin{array}{l}\text { Você acha que participar de um grupo de idos } \\
\text { bui para manutenção de sua qualidade de vid }\end{array}$ & $\begin{array}{l}\text { Verificar se o idoso pensa que a participação no grupo } \\
\text { contribui para a manutenção da sua qualidade de vida } \\
\text { e por quais motivos. }\end{array}$ \\
\hline $\begin{array}{l}\text { Você acha que idos } \\
\text { são menos depress }\end{array}$ & $\begin{array}{l}\text { Verificar se o idoso pensa que a participação no } \\
\text { grupo contribui para diminuir a propensão a sintomas } \\
\text { depressivos e por quais motivos. }\end{array}$ \\
\hline $\begin{array}{l}\text { O que você entende por depressão? Você sabe qual } \\
\text { a diferença entre depressão e tristeza? Ou trata-se da } \\
\text { mesma coisa? }\end{array}$ & $\begin{array}{l}\text { Verificar a concepção de depressão e tristeza que o } \\
\text { entrevistado possui e se ele sabe diferenciá-las. }\end{array}$ \\
\hline
\end{tabular}


Para análise dos dados quantitativos, utilizou-se estatística descritiva, sendo os dados apresentados na forma de média e desvio padrão (média $\pm \mathrm{DP}$ ) e/ou percentual (\%). Para análise das entrevistas, utilizou-se a técnica de análise em profundidade de Bardin (1979). Inicialmente, as entrevistas foram transcritas; após, foi realizada uma leitura global das repostas, com posterior leitura para identificação de ideias e, por fim, leitura para a categorização das falas das idosas.
Resultados

Ao final do trabalho, foram avaliadas 42 idosas que participam de grupos de terceira idade no município de Uruguaiana - RS. O grupo apresentou uma idade média de $71,3( \pm 5,8)$ anos, sendo a idade mínima de 60 anos e a máxima, 85 anos. O tempo médio de participação no grupo voltado à terceira idade foi de $3,6( \pm 2,8)$ anos. As características da amostra são apresentadas na Tabela 2.

Tabela 2. Características da amostra

\begin{tabular}{lr}
\hline \multicolumn{1}{c}{ Características sociodemográficas } & Estatísticas \\
\hline Idade (anos) & $71,3 \pm 5,8$ \\
Escolaridade (anos) & $5,3 \pm 4,1$ \\
Tempo de participação no grupo (anos) & $3,6 \pm 2,8$ \\
Estado civil & \\
Solteira & $7,1 \%(\mathrm{n}=3)$ \\
Casada & $21,4 \%(\mathrm{n}=9)$ \\
Viúva & $64,2 \%(\mathrm{n}=27)$ \\
Outros & $7,3 \%(\mathrm{n}=3)$ \\
Ocupação & \\
Aposentada & $50 \%(\mathrm{n}=22)$ \\
Dona de casa & $23,8 \%(\mathrm{n}=10)$ \\
Outros & $26,2 \%(\mathrm{n}=10)$ \\
Presença de patologias & \\
Hipertensão arterial sistêmica (HAS) & $40,5 \%(\mathrm{n}=17)$ \\
Não apresentam & $21,4 \%(\mathrm{n}=9)$ \\
Outros & $38,1 \%(\mathrm{n}=16)$ \\
\hline
\end{tabular}

Segundo os dados coletados, a maioria das idosas avaliadas tem preferência por praticar atividades físicas, como a ginástica, dentre as diferentes modalidades de atividades realizadas pelos grupos (musicoterapia, viagens e pas- seios) duas vezes por semana, uma hora por dia. Esses dados, juntamente com as características de participação nas atividades dos grupos de terceira idade, são expostos na Tabela 3 . 
Tabela 3. Características da amostra em relação à participação nas atividades do grupo

\begin{tabular}{lr}
\hline \multicolumn{2}{c}{ Atividades de maior interesse } \\
\hline Atividades físicas (ginástica) & $92,8 \%(\mathrm{n}=39)$ \\
Viagens & $4,8 \%(\mathrm{n}=2)$ \\
Musicoterapia & $2,4 \%(\mathrm{n}=1)$ \\
Número de atividades semanais realizadas pela maioria das idosas & 3 \\
Modalidade de atividades disponíveis semanalmente & $3,07 \pm 0,83$ \\
Tempo de atividades semanais (h) & $3,45 \pm 1,29$ \\
\hline
\end{tabular}

Com o objetivo de avaliar a função cognitiva geral, utilizou-se o MEEM. Nessa avaliação, verificou-se que as idosas apresentaram escore final médio de $24,0( \pm 3,03)$ pontos, tendo $16,6 \%$ delas ficado abaixo do ponto de corte para sua

Tabela 4. Características cognitivas da amostra de acordo com o MEEM e o EGD-30 escolaridade. Esse percentual de idosos apresenta características compatíveis com presença de deficit cognitivo. Os resultados de cada categoria avaliada pelo MEEM estão expressos na Tabela 4.

\begin{tabular}{lc}
\hline \multicolumn{1}{c}{ Instrumento/Categoria } & Estatísticas \\
\hline MEEM - escore total (30 pontos) & $24,04 \pm 3,03$ \\
MEEM - orientação (10 pontos) & $9,11 \pm 0,91$ \\
MEEM - registro (3 pontos) & $2,61 \pm 0,53$ \\
MEEM - cálculo (5 pontos) & $2 \pm 2,07$ \\
MEEM - evocação (3 pontos) & $2,11 \pm 0,88$ \\
MEEM - linguagem (9 pontos) & $8,5 \pm 0,73$ \\
MEEM - idosos abaixo do ponto de corte & $16,6 \%(\mathrm{n}=7)$ \\
EGD-30 (10 pontos) & $6,07 \pm 2,49$ \\
EGD idosos depressivos (n) & $11,9 \%(\mathrm{n}=5)$ \\
EGD idosos com depressão moderada $(n)$ & $11,9 \%(\mathrm{n}=5)$ \\
EGD idosos com depressão grave (n) & $0 \%(\mathrm{n}=0)$ \\
\hline
\end{tabular}


As idosas também foram avaliadas em relação à presença de depressão, por meio da EGD-30. Verificou-se que 88,1\% não apresentaram sintomas depressivos, $11,9 \%$ apresentaram sintomas de depressão moderada e nenhuma idosa apresentou depressão grave.

A qualidade de vida das participantes foi avaliada com base no SF-36. Constatou-se que as idosas apresentaram uma média de 98,6 $( \pm 6,22)$ pontos no escore total, sendo 111 a pontuação máxima possível. O SF-36 permite a avaliação da qualidade de vida considerando oito diferentes domínios, cujo escore individual pode variar de 0 a 100 . Os resultados obtidos nos diferentes domínios estão detalhados na Tabela 5.

Tabela 5. Avaliação da Qualidade de Vida (SF-36)

\begin{tabular}{lc}
\hline \multicolumn{1}{c}{ Domínio } & Média \pm DP \\
\hline Capacidade funcional & $75,9 \pm 15,7$ \\
Limitação por aspectos físicos & $75,5 \pm 38,4$ \\
Dor & $35,7 \pm 18,4$ \\
Estado geral de saúde & $51,5 \pm 12,8$ \\
Aspecto social & $54,7 \pm 12,3$ \\
Vitalidade & $54,5 \pm 17,6$ \\
Saúde mental & $55,7 \pm 19,8$ \\
Limitação dos aspectos emocionais & $70,6 \pm 34,6$ \\
\hline
\end{tabular}

Com base nas entrevistas semiestruturadas, também foi possível identificar a percepção das idosas acerca da qualidade de vida e dos fatores que a influenciam. Após leitura e categorização, percebeu-se a predominância das seguintes categorias: "A qualidade de vida está relacionada com aspectos físicos, tais como boa alimentação e prática de atividades físicas"; "A qualidade de vida está relacionada com a realização de atividades de lazer, como a participação em grupos de terceira idade"; "A fé em Deus e a proximidade dos familiares influenciam na qualidade de vida".

a) A qualidade de vida está relacionada com aspectos físicos, tais como boa alimentação e prática de atividades físicas

Ficou clara, nas falas das idosas, a predominância da concepção de qualidade de vida relacionada aos hábitos alimentares saudáveis e à prática de atividade física, como se pode perceber nas falas abaixo:

"Acredito que seja (qualidade de vida) ter uma vida feliz e comer coisas saudáveis, sem comer gorduras, sem fumar, e [...] fazer exercícios.” (M. L. P., 61 anos).

"Acho que é ter uma vida com hábitos saudáveis (pausa), como se exercitar, comer alimentos saudáveis, sem gorduras e ter uma boa saúde.”(C. Q., 77 anos).

Poucos idosos $(8,19 \%)$ citaram que a qualidade de vida está relacionada ao fato de terem independência para realizarem suas atividades cotidianas, como na seguinte fala:

“[...] por qualidade eu entendo que é ter uma liberdade com tudo, com os amigos, com as [...] fazer bem para todo mundo, a gente ter independência pra ir nos vizinhos, pra tomar banho, ir no mercado, limpar a casa (pausa) essas coisas no mais.” (R. F., 71 anos).

b) A qualidade de vida está relacionada com a realização de atividades de lazer, como a participação em grupos de terceira idade 
Muitos idosos relataram que ter um lazer, como a participação no grupo de terceira idade, e não ficar na rotina, além de praticar atividade física e manter uma alimentação saudável, são fatores que influenciam em sua qualidade de vida, como evidencia a seguinte fala:

"Acho que [ter qualidade de vida] são as atividades de dançar, como no grupo, fazer ginástica e conversar e também [...] comer alimentos saudáveis e sair da rotina, tu sabe que esse grupo nos ajuda e nos motiva a sair de casa porque aqui esquecemos do que está acontecendo lá fora, coisa bem boa (risos)!" (N. A., 69 anos).

Em relação à participação no grupo de terceira idade, foram apontados muitos pontos positivos, os quais influenciam diretamente na manutenção da qualidade de vida.

"Estar nesse grupo nos ajuda e muito, porque aqui fazemos ginástica e [...] temos palestras que nos deixam mais informadas, e também é bom porque a gente tira as dúvidas [...], a gente dança e se diverte com as companheiras, a gente esquece dos problemas lá fora.” (E. O., 68 anos).

"Sim, e como influencia (a participação no grupo influencia a qualidade de vida)! Aqui passa as horas mais rápido, parece, durante a semana, a gente fica contando pra que passe os dias duma vez pra que chegue aquele dia [...] de estar aqui, eu sou assim, até minhas dores sumiram depois que comecei a participar aqui, pelo menos desde que eu entrei me sinto muito bem.” (I. C., 76 anos).

A maioria das idosas ressaltou o fato de que pessoas que participam de grupos de terceira idade apresentam menor incidência de depressão:
"Acho que sim [quem participa do grupo tem menor probabilidade de sentir-se depressivo]. Ah, porque saem mais de casa, não ficam só na frente da TV, como muitas amigas que tenho, e ocupam a cabeça com coisas saudáveis.” (T. M., 81 anos).

"Ah, com certeza quem participa do grupo fica menos depressivo! Porque aqui temos palestras que nos ajudam a entender muita coisa [...], e temos oficinas pra nos distrair a cabeça e fazemos exercícios [...]. É maravilhoso pra o nosso corpo e a nossa cabeça." (E. L., 67 anos).

Algumas idosas souberam diferenciar depressão e tristeza, porém a maioria mostrou-se confusa, apontando ideias contraditórias em suas falas, uma vez que relatavam que depressão e tristeza têm o mesmo significado:

“[...] já ouvi falarem disso [depressão], mas não sei dizer bem [...] é uma doença que atrai pensamentos ruins, assim como a tristeza, acho que é isso.” (M. S., 71 anos).

“É uma tristeza, eu imagino [a depressão], não sei, nunca tive, eu vejo falar que as pessoas sentem uma tristeza, uma agonia, uma ansiedade, coisa assim (pausa). Ah, não sei, nunca tive. Quase a mesma coisa [tristeza e depressão], porque [...] a pessoa com depressão fica desnorteada, né? Tristeza é ficar abalado, ficar triste, depressão não tem vontade de nada, pelo menos as pessoas que eu conheço querem estar sozinhas, encerradas. Apesar de tudo eu nunca tive, graças a Deus.” (A. B., 75 anos).

c) A fé em Deus e a proximidade dos familiares influenciam na qualidade de vida

Pôde-se perceber que foi recorrente na fala das idosas a ideia de que estar próximo aos familiares e ter fé em Deus influencia na qualidade de vida: 
"Ah [...] sair de casa, conversar com as amigas, estar perto da família, dos filhos e netos, ainda mais a gente que perdeu o marido cedo (pausa), acho que rezar bastante e pedir a proteção divina sempre ajuda também [a ter qualidade de vida], a gente se fortalece tendo fé em Deus." (N. S., 72 anos).

"Acho que é olhar pra trás e ver quantas coisas boas construímos e poder ter os filhos, netos todos por perto e também conversar com eles e contar um pouco da nossa vida antigamente, não era fácil. $\mathrm{E}$ essas coisas que acontecem na nossa vida são graças a nossa fé, em ter fé e pedir pela saúde de todos que tão ao nosso redor.” (C. L., 69 anos).

\section{Discussão}

Neste estudo, foram avaliadas a função cognitiva geral, a incidência de depressão e a qualidade de vida de 42 idosas que participam de grupos de terceira idade de Uruguaiana - RS, bem como sua percepção acerca desses aspectos.

Observou-se que o desempenho cognitivo da minoria das idosas avaliadas apresentou características compatíveis com presença de deficit cognitivo. As idosas evidenciaram um escore final de 24 $( \pm 3,03)$ pontos no MEEM, tendo $16,6 \%$ delas ficado abaixo do ponto de corte para sua escolaridade, considerando a classificação proposta por Kochhann et al. (2010). Esse percentual de idosas requer atenção e cuidados médicos, os quais serão adequadamente recomendados à família. Uma vez que a maioria das idosas avaliadas demonstrou ter função cognitiva preservada, pressupõe-se que esse aspecto possa estar relacionado à participação no grupo de idosos e à rea- lização das atividades cognitivas, recreativas e físicas que esta envolve.

Esses resultados estão de acordo com a hipótese inicial e, também, com a literatura da área. Alguns estudos sugerem que idosos com função cognitiva preservada têm estilo de vida mais saudável, são mais ativos e apresentam melhor capacidade funcional. (MCGUIRE; FORD; AJANI, 2006; BASSUK; WYPIJ; BERKMAN, 2000). Além disso, dados epidemiológicos sugerem que pessoas moderadamente ativas têm menor risco de ser acometidas por desordens mentais do que as sedentárias, mostrando que indivíduos fisicamente ativos, provavelmente, possuem um processamento cognitivo mais rápido. (ANTUNES et al., 2006). Portanto, nossas idosas, possivelmente devido ao seu estilo de vida e à participação em grupos de terceira idade, que promovem práticas de atividades físicas e cognitivas, bem como convívio social intenso, apresentam uma boa função cognitiva geral.

Com relação à incidência de depressão, verificou-se que $88,1 \%$ das idosas avaliadas não apresentam sintomas depressivos e $11,9 \%$ apresentam sintomas de depressão moderada. Estudos prévios mensuraram a incidência de depressão em idosos com diferentes características e evidenciam que idosos não participantes de grupos tendem a apresentar maior incidência de depressão. Nardi e Andrade (2005) avaliaram a depressão em uma comunidade do Sul do Brasil, no estado do Paraná, e verificaram que $28,8 \%$ dos idosos apresentaram depressão moderada. Silva et al. (2011) avaliaram um grupo de idosos institucionalizados de 
Uruguaiana - RS e verificaram que $60 \%$ dos idosos evidenciaram características depressivas. Já Oliveira, Gomes e Oliveira (2006), ao estudarem idosos frequentadores de grupos de convivência, encontraram depressão em $31 \%$ deles. Assim, nossas idosas evidenciam índices de depressão menores do que os divulgados pela maioria das pesquisas, isso porque, do ponto de vista psicológico, as atividades físicas e recreativas e o convívio em grupo podem ajudar no combate à depressão, atuando como catalisadoras de relacionamento interpessoal e estimulando a autoestima pela superação de pequenos desafios. (ANTUNES et al., 2001).

$\mathrm{Na}$ avaliação da qualidade de vida, apesar da inexistência de pontos de corte na escala do SF-36, é lícito interpretar que valores acima de 60/70 pontos em cada um dos domínios avaliados, em uma escala de 0 a 100, indiquem uma qualidade de vida preservada ou boa. (CAMPOS; PORTO, 2009). Embora em alguns domínios os resultados tenham beirado o limite inferior, pode-se considerar que a maioria das idosas apresenta uma qualidade de vida, quando associados os resultados do SF-36 com os obtidos nas entrevistas qualitativas. O SF-36 também permitiu verificar que o fator que parece ser mais prejudicial à qualidade de vida das idosas é a dor.

O envelhecer com qualidade de vida, de acordo com a percepção das idosas entrevistadas, está estreitamente relacionado com aspectos físicos, tais como boa alimentação e prática de atividades físicas. A nutrição, a saúde e o envelhecimento estão vinculados entre si; logo, a manutenção de uma dieta equilibrada está realmente associada a um envelhecimento saudável. (PESSOA, 2012). Já a prática de atividade física regular, como a que normalmente ocorre com a participação em grupos de terceira idade, gera maior grau de motivação e maior sentimento de autoeficácia, diminuindo a probabilidade de desenvolvimento de doenças crônicas e melhorando a aptidão física, disposição geral e, consequentemente, a qualidade de vida. (FERREIRA; MARINS; SILVA, 2006).

Um dos pontos fortes do trabalho foi a associação feita pelas idosas em suas falas entre a participação no grupo e a boa qualidade de vida que apresentam, validando nossa principal hipótese. A segurança oferecida por um ambiente acolhedor e estimulador, como o dos grupos, propicia autonomia ao indivíduo idoso, necessária ao seu bem-estar. (PENNA; SANTOS, 2006). Mello (2005) também destaca que as atividades de lazer e a convivência em grupo contribuem tanto para a manutenção do equilíbrio biopsicossocial dos idosos, como para o ritmo biológico, o sono, a memória, funções muitas vezes alteradas devido ao processo de envelhecimento.

Por fim, como evidenciam as falas das idosas deste estudo e como relata a literatura, a fé em Deus e a proximidade dos familiares também influenciam na qualidade de vida. (BARRICELLI et al., 2012). A correlação da religiosidade e da qualidade de vida deve-se ao fato de este último valor ter um conceito multidimensional, que envolve aspectos físicos, sociais e emocionais. 


\section{Conclusão}

O envelhecimento, apesar de tratar-se de um fenômeno natural, geralmente é associado a diversos fatores negativos. Para que seja possível promover um envelhecimento saudável, um dos aspectos importantes é a manutenção da autonomia e da qualidade de vida. Este estudo permite verificar que a participação dos idosos em grupos de convivência revela-se como uma alternativa para a manutenção das funções cognitivas, a diminuição da incidência de depressão e o aumento da qualidade de vida dessa população. O convívio social, o sentimento de autonomia e a prática de atividades físicas e recreativas proporcionadas por esses grupos contrapõem-se aos principais problemas enfrentados por essa parcela da população, e as idosas entrevistadas têm uma percepção clara dessa importância, colocando o grupo como um dos principais fatores relacionados à sua qualidade de vida.

Embora os resultados positivos aqui relatados sejam semelhantes aos de outros estudos, novas abordagens do tema são necessárias. Com o delineamento de natureza transversal aqui utilizado, tornam-se restritas as inferências em relação ao impacto que a participação nas atividades do grupo tem na qualidade de vida dos idosos e na incidência de depressão. Também, a inserção de um grupo controle, composto por idosas não participantes de grupos de terceira idade, é um aspecto importante para melhor compreensão dos efeitos desse tipo de intervenção.
Quality of life and incidence of depression in older women that attend groups of elderly

\section{Abstract}

The objective of this study was to evaluate the quality of life and the incidence of depression in elderly people that attend groups of elderly of Uruguaiana/RS and relate these results with the perception of the elderly about these aspects. 42 elderly women participants of elderly's groups of Uruguaiana/RS were selected by. The elderly were evaluated about her cognition (Mini-Mental State Examination, MMSE), presence of depression (Geriatric Depression Scale, GDS-30) and quality of life (SF36), and interviewed with the objective of verify their perception about the same aspects. The quantitative results were by descriptive statistic; and, the interviews were recorded and subsequently transcribed for a depth analysis. The average age of the sample was 71.3 years, with average time of participation in the group of 3.6 years. The average score in the MMSE was 24.04 points, with only 7 elderly presented a score below the cutoff point. In GDS-30 we veirfy that $88.1 \%$ of elderly did not show depressive symptoms and 11.9 showed symptoms of moderate depression. The average of the SF-36 was 98.6, which, along with the ideas present in the orderly's speeches, shows that most of elderly have a good quality of life. We found that the incidence of depression and cognitive deficit among the elderly evaluated was low and the elderly have a good quality of life, which may be related with their participation in living groups, as emphasized in their speeches.

Keywords: Quality of life. Mini-Mental. Physical activity. Elderly. Seniors. 


\section{REFERÊNCIAS}

ANTUNES, H. K. M. et al. Alterações cognitivas em idosas decorrentes do exercício físico sistematizado. Revista da Sobama, São Carlos, v. 6, n. 1, p. 27-33, dez. 2001.

ANTUNES, H. K. M. et al. Reviewing on physical exercise and the cognitive function. Revista Brasileira de Medicina do Esporte, Niterói, v. 12, n. 2, p. 108-114, mar./abr. 2006.

BASSUK, S. S.; WYPIJ, D.; BERKMAN, L. F. Cognitive impairment and mortality in the community-dwelling elderly. American Journal of Epidemiology, v. 151, n. 7, p. 676-688, 2000.

BARDIN, L. Análise de conteúdo. Lisboa: Persona, 1979.

BARRICELLI, I. L. F. O. B. L. et. al. Influência da orientação religiosa na qualidade de vida de idosos ativos. Revista Brasileira de Geriatria e Gerontolologia, Rio de Janeiro, v. 15, n. 3, p. 505-515, 2012.

CAMPOLINA, A. G.; CICONELLI, R. M. O SF-36 e o desenvolvimento de novas medidas de avaliação de qualidade de vida. Acta Reumatológica Portuguesa, Algés, v. 33, n. 2, p. 127-133. 2008.

CAMPOS, F. V. S.; PORTO, L. G. G. Qualidade de vida e nível de atividade física de pacientes em fase ambulatorial da reabilitação cardíaca. Revista Brasileira de Atividade Física \& Saúde, Pelotas, v. 14, n. 2, p. 86-95, 2009.

CASAGRANDE, M. Atividade Física na Terceira Idade. 2006. 56 f. Dissertação (Mestrado em Educação Física) - Universidade Estadual Paulista, Bauru, São Paulo, 2006.

FERREIRA, M. et al. Efeitos de um programa de orientação de atividade física e nutricional sobre o nível de atividade física de mulheres fisicamente ativas de 50 a 72 anos de idade. Revista Brasileira de Medicina do Esporte, São Paulo, v. 11, n. 3, p. 172-176, maio/jun. 2005.
FERREIRA, S.; MARINS, J. C. B.; SILVA, L. C. Determinação de perfil de repetições máximas no exercício de extensão de pernas e supino reto com diferentes percentuais de força. Revista de Educação Física, Maringá, v. 17, n. 2, p. 149-159, 2006.

FREIRE, S. A. Envelhecimento bem-sucedido e bem-estar psicológico. In: NERI, A. L.; FREIRE, S. A. (Orgs.). E por falar em boa velhice. Campinas: Papirus, 2000. p. 21-31.

FRUTUOSO, D. A terceira idade na universidade. Rio de Janeiro: Ágora da Ilha, 1999.

KATZ, L.; RUBIN, M. Mantenha o seu cérebro vivo. São Paulo: Sextante, 2000.

KOCHHANN, R. et al. The Mini-Mental State Examination: review of cutoff points adjusted for schooling in a large Southern Brazilian sample. Dementia Neuropsychologia, São Paulo, v. 4, n. 1, p. 35-44, Mar. 2010.

MCGUIRE, L. C.; FORD, E. S.; AJANI, U. A. The impact of cognitive functioning on mortality and the development of functional disability in older adults with diabetes: the second longitudinal study on aging. $B M C$ Geriatrics, London, v. 6, n. 8, p. 8, 2006.

MARQUES-TEIXEIRA, J. Depressão e a mulher na sociedade moderna. Psiquiatria em Revista, Rio de Janeiro, v. 11, n. 3, p. 5-54, 1998.

MENDES, M. R. S. S. B. et al. A situação social do idoso no Brasil: uma breve consideração. Acta Paulista de Enfermagem, São Paulo, v. 18, n. 4, p. 422-426, 2005.

MELLO, M. T. O. O exercício físico e os aspectos psicobiológicos. Revista Brasileira de Medicina do Esporte, São Paulo, v. 11, n. 3, p. 203-207, maio/jun. 2005.

NASCIMENTO, M. C. R. et al. Qualidade de vida na terceira idade. In: PAES, S. et al. Envelhecer com cidadania: quem sabe um dia? Rio de Janeiro: ANG, 2000. p. 121-137. 
NARDI, E. F. R.; ANDRADE, O. G. de. Estados depressivos entre idosos na comunidade - Jandaia do Sul, Paraná, Brasil. Arquivos de Ciências da Saúde Unipar, Umuarama, v. 9, n. 2, p. 109-116, maio/ago. 2005.

ODEBRECH, T. A. C.; PEDROSO, R. Qualidade de vida no trabalho: diferentes percepções de um mesmo processo. Revista Olhar Científico, Ariquemes, v. 1, n. 1, p. 134-153, ago./dez. 2010.

OLIVEIRA, D. A. A. P.; GOMES, L.; OLIVEIRA, R. F. Prevalência de depressão em idosos que frequentam centros de convivência. Revista de Saúde Pública, São José dos Campos, v. 40, n. 4, p. 734-736, 2006.

PALMA, L. T. S. Educação permanente e qualidade de vida: indicativos para uma velhice bem-sucedida. Passo Fundo: UPF Editora, 2000.

PENNA, F. B.; SANTOS, F. H. E. O movimento das emoções na vida dos idosos: um estudo com um grupo da terceira idade. Revista Eletrônica de Enfermagem, São Gonçalo, $v$. 8, n. 1, p. 17-24, 2006.

PESSOA, L. M. O. Adequação nutricional da alimentação servida aos idosos do Instituto São Vicente de Paulo: vinculado ao laboratório itinerante- PROEAC/UEPB. 2012. $39 \mathrm{f}$. Trabalho de Conclusão de Curso (Bacharelado em Enfermagem) - Universidade Estadual da Paraíba, Campina Grande, PB, 2012.

RIBARIC, S. Diet and aging. Oxidative Medicine and Cellular Longevity, New York, p. 1-20, 2012. Doi:10.1155/2012/741468.

SILVA, A. O. et al. Perfil cognitivo de idosos institucionalizados de Uruguaiana/RS. $R e$ vista Contexto \& Saúde, Ijuí, v. 10, n. 20, p. 1185-1190, jan./jul. 2011.

SILVA, M. N. M. et al. Depressão em mulheres climatéricas: análise de mulheres atendidas ambulatorialmente em um hospital universitário no Maranhão. Revista de Psiquiatria do RS, Porto Alegre, v. 30, n. 2, p. 150-154, 2008.
TEIXEIRA, D. de C.; OKUMA, S. S. Efeitos de um programa de intervenção para idosos sobre a intenção de estudantes de educação física de trabalhar com este grupo etário. Revista Brasileira de Educação Física e Esporte, São Paulo, v. 18, n. 2, p. 137-149, 2004.

WORLD HEALTH ORGANIZATION. Envelhecimento ativo: uma política de saúde. Trad. de Suzana Gontijo. Brasília: Organização Pan-Americana da Saúde, 2005. 60 p.

YESAVAGE, J. A. et al. Development and validation of a geriatric depression screening scale: a preliminary report. Journal of Psychiatry Research, Stanford, v. 17, n. 1, p. 37-49, 1983. 